CRYSTALLOGRAPHIC COMMUNICATIONS

ISSN 2056-9890

Received 9 February 2016

Accepted 8 March 2016

Edited by H. Stoeckli-Evans, University of Neuchâtel, Switzerland

Keywords: crystal structure; redetermination; hydrogen bonding; refinement constraints; refinement restraints; Cambridge Structural Database.

CCDC reference: 1459296

Supporting information: this article has supporting information at journals.iucr.org/e

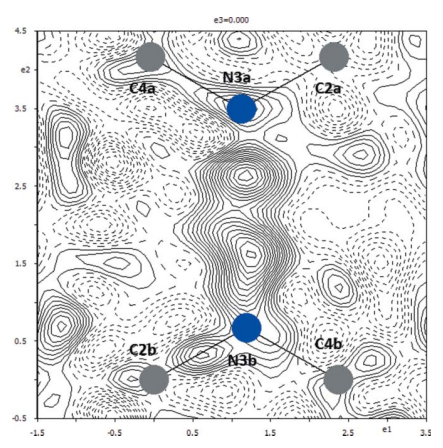

OPEN $\odot$ ACCESS

\section{Redetermination of cytosinium hydrogen maleate- cytosine (1/1) from the original data}

\author{
Jan Fábry*
}

Inst. of Physics of the Czech Academy of Sciences, Na Slovance 2, 18221 Praha 8, Czech Republic. *Correspondence e-mail: fabry@fzu.cz

The title salt, $\mathrm{C}_{4} \mathrm{H}_{6} \mathrm{~N}_{3} \mathrm{O}^{+} \cdot \mathrm{C}_{4} \mathrm{H}_{3} \mathrm{O}_{4}{ }^{-} \cdot \mathrm{C}_{4} \mathrm{H}_{5} \mathrm{~N}_{3} \mathrm{O}$, has been redetermined from the data published by Benali-Cherif, Falek \& Direm [Acta Cryst. (2009), E65, o3058-o3059]. The improvement of the present redetermination consists in the discovery of the splitting of one of the $\mathrm{H}$ atoms into two disordered positions, the occupancies of which are equal to 0.55 (2) and 0.45 (2). These H atoms are involved in an $\mathrm{N} \cdots \mathrm{N}$ hydrogen bond and are shifted towards its centre. The disorder of these $\mathrm{H}$ atoms is in agreement with a similar environment of the two independent, but chemically equivalent, cytosinium/cytosine molecules.

\section{Chemical context}

Structures which contain hydroxyl, secondary and primary amine groups are often determined incorrectly because of an assumed geometry of these groups and the subsequent applied constraints or restraints. In such cases, the correct geometry is missed as it is not verified by inspection of the difference electron-density maps. Thus a considerable number of structures could have been determined more correctly $-c f$. Figs. 1 and 2 in Fábry et al. (2014). The inclusion of such structures causes bias in the crystallographic databases.<smiles>Nc1cc[nH]c(=O)n1</smiles>

In the course of recalculation of suspect structures which were retrieved from the Cambridge Crystallographic Database (Groom \& Allen, 2014), a defect in the structure determination of 2-amino-4,6-dimethoxypyrimidine-4-aminobenzoic acid (1/1) by Benali-Cherif et al. (2009) has been found; the CSD refcode is DUJCAN. The aim of the present article is to demonstrate how the original structure determination can be improved.

\section{Structural commentary}

The structure of the title compound has been described by Benali-Cherif et al. (2009). In that article, the hydrogen atom $\mathrm{H} 3 b$ was attached to atom $\mathrm{N} 3 b$ and refined with a distance constraint of $\mathrm{N} 3 b-\mathrm{H} 3 b=0.86 \AA$ with $U_{\text {iso }}(\mathrm{H} 3 b)=$ $1.2 U_{\mathrm{eq}}(\mathrm{N} 3 b)$. This hydrogen is involved in the hydrogen bond $\mathrm{N} 3 b-\mathrm{H} 3 \mathrm{~b} \cdot \mathrm{N} 3 a$ (Fig. 1). 

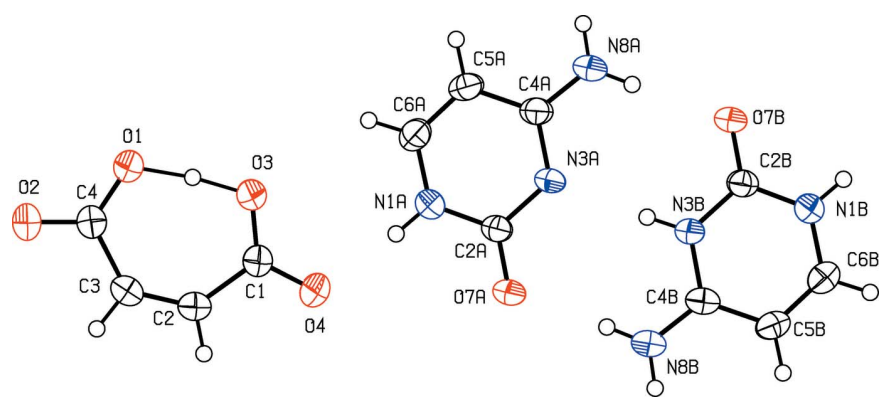

Figure 1

View of the constituent molecules and atoms of the title structure in the original article [Benali-Cherif, Falek \& Direm (2009). Acta Cryst. E65, o3058-03059]. The displacement ellipsoids are drawn at the $50 \%$ probability level.

However, inspection of the difference electron density map of the recalculated structure has shown that hydrogen atom $\mathrm{H} 3 b$ is disordered over two positions (Fig. 2), between atoms $\mathrm{N} 3 a$ and $\mathrm{N} 3 b$. Thus, atom $\mathrm{H} 3 b$ was split into two atoms, labelled as $\mathrm{H} 1 n 3 b$ and $\mathrm{H} 1 n 3 a$, with respective occupancies 0.52 (2) and 0.48 (2). These hydrogen atoms remain involved in the $\mathrm{N} 3 a \cdots \mathrm{N} 3 b$ hydrogen bond (Table 1), as shown in Fig. 3.

The observed disorder of the secondary amine hydrogen atoms is probably due to the chemical equality of two symmetry-independent cytosinium/cytosine molecules and their quite similar environments. Otherwise, the description of

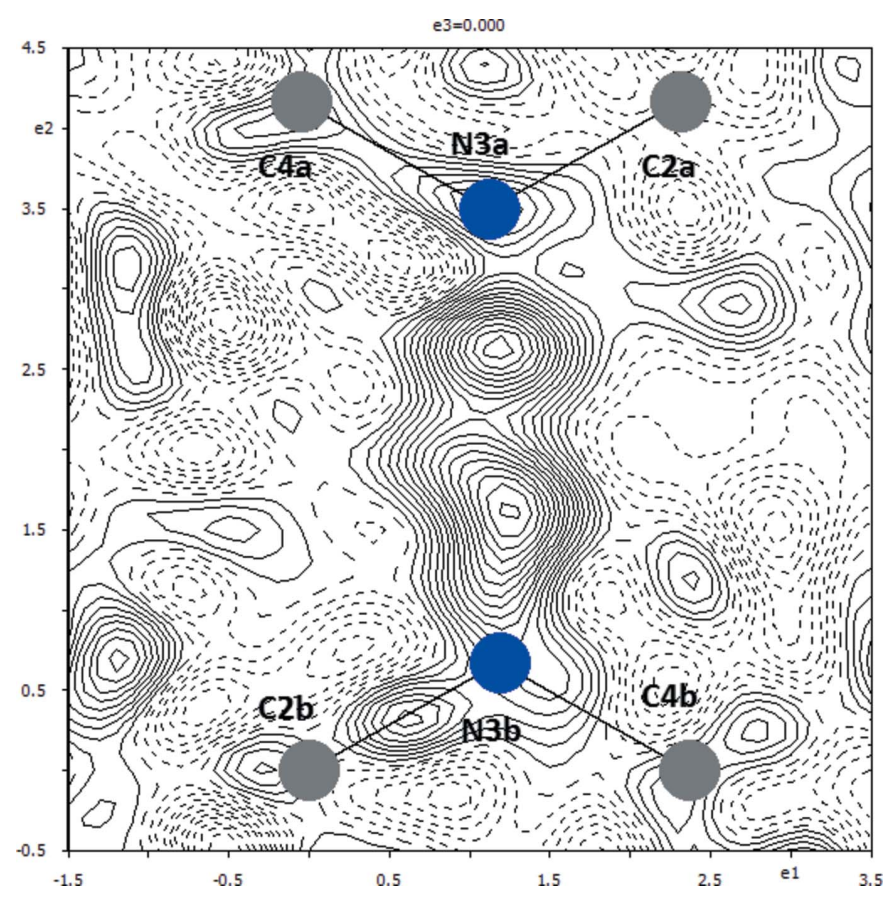

Figure 2

A section of the difference electron-density map for the present redetermined title structure, which shows the build up of the electron density between atoms N1 and N3. Positive and negative electron densities are indicated by continuous and dashed lines, respectively. The increment between the contours is $0.05 \mathrm{e}^{-3}$ (JANA2006; Petříček et al., 2014).
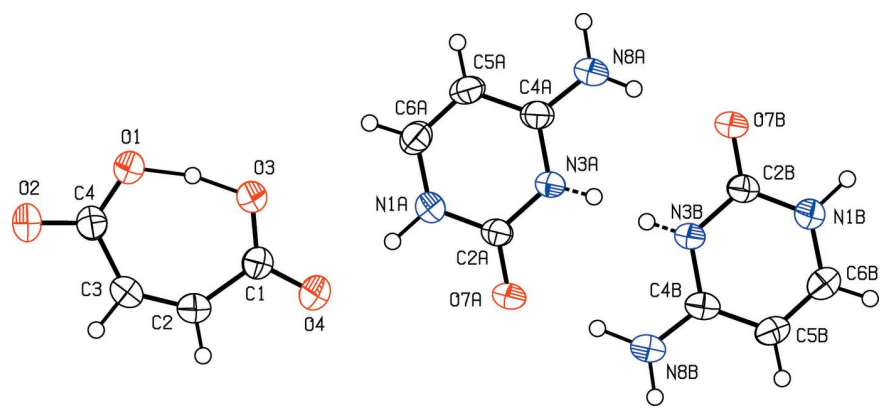

Figure 3

View of the constituent molecules and atoms of the present redetermined title structure. The displacement ellipsoids are drawn at the $50 \%$ probability level.

the hydrogen-bond pattern by Benali-Cherif et al. (2009) remains intact because locally one of the nitrogen atoms, N3a or $\mathrm{N} 3 b$, acts as a donor while the other acts as an acceptor of the hydrogen bond.

The hydrogen atom $\mathrm{H} 3$, which was situated about the centre of the hydrogen bond $\mathrm{O} 3-\mathrm{H} 3 \cdots \mathrm{O} 1$ has also been checked (Fig. 4). It turns out that the build-up of the electron density is not split into two positions and the original position determined by Benali-Cherif et al. (2009) is correct.

In a broader sense, the present redetermination emphasizes how important it is to carefully examine the difference electron-density maps during structure determinations.

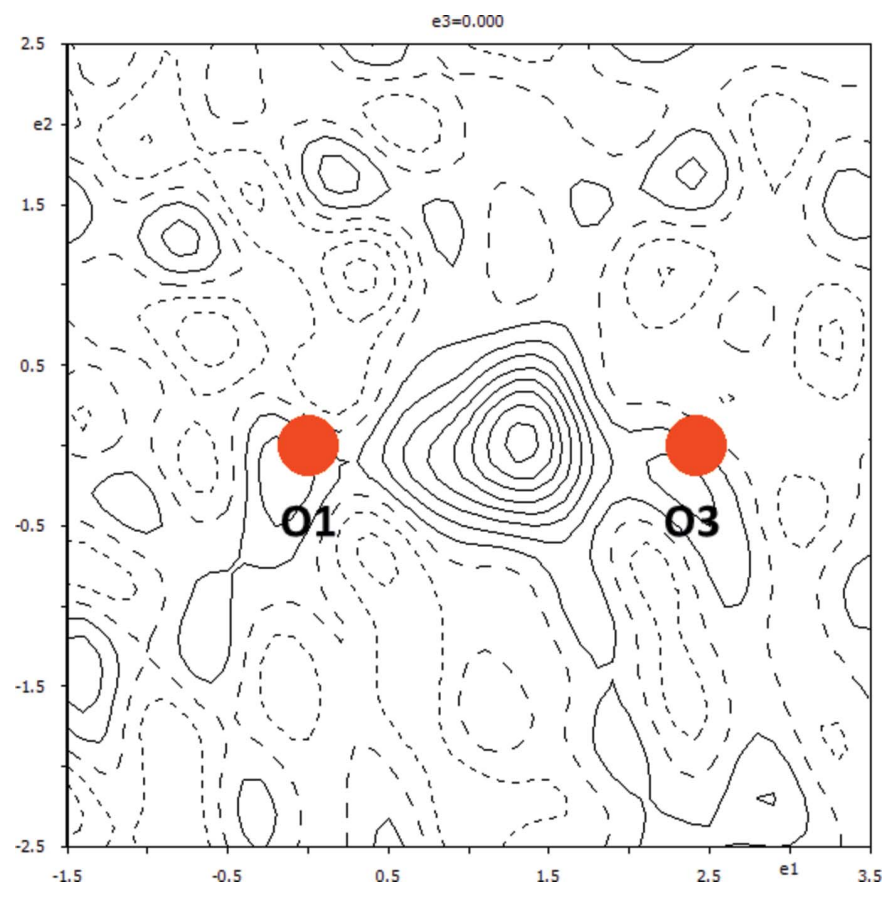

Figure 4

A section of the difference electron-density map for the present redetermined title structure, which shows the build up of the electron density between atoms $\mathrm{O} 1$ and $\mathrm{O} 3$. Positive and negative electron densities are indicated by continuous and dashed lines, respectively. The increment between the contours is $0.05 \mathrm{e} \AA^{-3}$ (JANA2006; Petř́íček et al., 2014). 
Table 1

Hydrogen-bond geometry $\left(\AA,^{\circ}\right)$.

\begin{tabular}{lllll}
\hline$D-\mathrm{H} \cdots A$ & $D-\mathrm{H}$ & $\mathrm{H} \cdots A$ & $D \cdots A$ & $D-\mathrm{H} \cdots A$ \\
\hline $\mathrm{N} 1 b-\mathrm{H} 1 b \cdots \mathrm{O} 2^{\mathrm{i}}$ & $0.956(15)$ & $1.824(15)$ & $2.7718(14)$ & $170.8(13)$ \\
$\mathrm{N} 8 b-\mathrm{H} 8 b 1 \cdots \mathrm{O} 7 b^{\mathrm{ii}}$ & $0.900(17)$ & $2.030(18)$ & $2.8517(14)$ & $151.2(13)$ \\
$\mathrm{N} 8 b-\mathrm{H} 8 b 2 \cdots \mathrm{O} 7 a$ & $0.992(15)$ & $1.850(15)$ & $2.8411(15)$ & $177.4(12)$ \\
$\mathrm{C} 5 b-\mathrm{H} 5 b \cdots \mathrm{O} 2^{\mathrm{iii}}$ & 0.93 & 2.43 & $3.3347(16)$ & 164.60 \\
$\mathrm{~N} 1 a-\mathrm{H} 1 a \cdots \mathrm{O} 4$ & $0.952(14)$ & $1.793(14)$ & $2.7411(14)$ & $173.2(12)$ \\
$\mathrm{N} 8 a-\mathrm{H} 8 a 1 \cdots \mathrm{O} 7 b$ & $0.897(16)$ & $1.959(16)$ & $2.8555(15)$ & $179.0(13)$ \\
$\mathrm{N} 8 a-\mathrm{H} 8 a 2 \cdots \mathrm{O} 7 a^{\text {iv }}$ & $0.885(17)$ & $2.028(18)$ & $2.8368(15)$ & $151.5(14)$ \\
$\mathrm{C} 5 a-\mathrm{H} 5 a \cdots \mathrm{O} 4^{\mathrm{i}}$ & 0.93 & 2.37 & $3.2970(16)$ & 175.15 \\
$\mathrm{O} 1-\mathrm{H} 3 \cdots \mathrm{O} 3$ & $1.223(14)$ & $1.201(14)$ & $2.4155(12)$ & $170.6(15)$ \\
$\mathrm{O} 1-\mathrm{H} 3 \cdots \mathrm{C} 1$ & $1.223(14)$ & $2.071(15)$ & $3.0775(15)$ & $136.7(11)$ \\
$\mathrm{O} 3-\mathrm{H} 3 \cdots \mathrm{O} 1$ & $1.201(14)$ & $1.223(14)$ & $2.4155(12)$ & $170.6(15)$ \\
$\mathrm{O} 3-\mathrm{H} 3 \cdots \mathrm{C} 4$ & $1.201(14)$ & $2.100(15)$ & $3.0927(15)$ & $137.4(12)$ \\
$\mathrm{N} 3 b-\mathrm{H} 1 n 3 b \cdots \mathrm{N} 3 a$ & $0.861(16)$ & $1.979(16)$ & $2.8398(14)$ & $178(2)$ \\
$\mathrm{N} 3 a-\mathrm{H} 1 n 3 a \cdots \mathrm{N} 3 b$ & $0.873(18)$ & $1.970(18)$ & $2.8398(14)$ & $174(3)$ \\
\hline
\end{tabular}

Symmetry codes: (i) $x+\frac{1}{2},-y+\frac{3}{2}, z-\frac{1}{2}$; (ii) $x, y-1, z$; (iii) $x+\frac{1}{2},-y+\frac{1}{2}, z-\frac{1}{2}$; (iv) $x, y+1, z$.

\section{Supramolecular features}

The graph set analysis (Etter et al., 1990) of the title compound has been described by Benali-Cherif et al. (2009).

\section{Database survey}

The CIF file of the article by Benali-Cherif et al. (2009) has been included in the Cambridge Crystallographic Database (Groom \& Allen, 2014) under the refcode DUJCAN.

\section{Synthesis and crystallization}

The preparation of the title compound has been described by Benali-Cherif et al. (2009).

\section{Refinement}

Crystal data, data collection and structure refinement details are summarized in Table 2. All the hydrogen atoms were discernible in the difference electron density maps. The aryl hydrogen atoms were refined as constrained with $\mathrm{C}_{\text {aryl }}-\mathrm{H}_{\text {aryl }}$ $=0.93 \AA$ and $U_{\text {iso }}\left(\mathrm{H}_{\text {aryl }}\right)=1.2 U_{\text {eq }}\left(\mathrm{C}_{\text {aryl }}\right)$. The displacement parameter of the hydroxyl hydrogen atom $\mathrm{H} 3$ was constrained by $U_{\text {iso }}(\mathrm{H} 3)=1.5 U_{\text {eq }}(\mathrm{O} 3)$. The hydrogen atoms of the primary and secondary amine groups were constrained by $U_{\text {iso }}\left(\mathrm{H}_{\text {amine }}\right)$ $=1.2 U_{\text {eq }}\left(\mathrm{N}_{\text {amine }}\right)$. In addition, the distances of the disordered amine hydrogen atoms, $\mathrm{H} 1 n 36$ and $\mathrm{H} 1 n 3 b$, were refined with the distance restraint $\mathrm{N}-\mathrm{H}=0.87$ (1) $\AA$, and their occupational parameters constrained to fulfill the condition that their sum $=1$ [viz. $0.55(2)(\mathrm{H} 1 n 3 b)$ and $0.45(2)(\mathrm{H} 1 n 3 \mathrm{a})]$.

Nine reflections [5 $10 ;-911 ;-111 ;-821 ; 421 ;-202 ; 00$ 2;-3 12 2; -2008 8; 2228 8] for which $\left\|F_{\mathrm{o}}|-| F_{\mathrm{c}}\right\|>10 \sigma(F)$ were omitted from the final cycles of refinement.
Table 2

Experimental details.

\begin{tabular}{|c|c|}
\hline \multicolumn{2}{|l|}{ Crystal data } \\
\hline Chemical formula & $\mathrm{C}_{4} \mathrm{H}_{6} \mathrm{~N}_{3} \mathrm{O}^{+} \cdot \mathrm{C}_{4} \mathrm{H}_{3} \mathrm{O}_{4}{ }^{-} \cdot \mathrm{C}_{4} \mathrm{H}_{5} \mathrm{~N}_{3} \mathrm{O}$ \\
\hline$M_{\mathrm{r}}$ & 338.29 \\
\hline Crystal system, space group & Monoclinic, $C 2 / c$ \\
\hline Temperature (K) & 298 \\
\hline$a, b, c(\AA)$ & $27.3226(5), 7.3618(2), 14.6742(4)$ \\
\hline$\beta\left(^{\circ}\right)$ & $93.905(1)$ \\
\hline$V\left(\AA^{6}\right)$ & $2944.77(13)$ \\
\hline$Z$ & 8 \\
\hline Radiation type & Мo $K \alpha$ \\
\hline$\mu\left(\mathrm{mm}^{-1}\right)$ & 0.13 \\
\hline Crystal size $(\mathrm{mm})$ & $0.3 \times 0.15 \times 0.1$ \\
\hline \multicolumn{2}{|l|}{ Data collection } \\
\hline Diffractometer & Nonius KappaCCD \\
\hline $\begin{array}{l}\text { No. of measured, independent and } \\
\text { observed }[I>3 \sigma(I)] \text { reflections }\end{array}$ & $3490,3474,2367$ \\
\hline$R_{\text {int }}$ & 0.043 \\
\hline$(\sin \theta / \lambda)_{\max }\left(\AA^{-1}\right)$ & 0.661 \\
\hline \multicolumn{2}{|l|}{ Refinement } \\
\hline$R\left[F^{2}>3 \sigma\left(F^{2}\right)\right], w R\left(F^{2}\right), S$ & $0.038,0.093,1.85$ \\
\hline No. of reflections & 3474 \\
\hline No. of parameters & 246 \\
\hline No. of restraints & 2 \\
\hline H-atom treatment & $\begin{array}{l}\mathrm{H} \text { atoms treated by a mixture of } \\
\text { restrained and constrained } \\
\text { refinement }\end{array}$ \\
\hline$\Delta \rho_{\max }, \Delta \rho_{\min }\left(\mathrm{e} \AA^{-3}\right)$ & $0.20,-0.20$ \\
\hline
\end{tabular}

Computer programs: KappaCCD Server Software (Nonius, 1998), DENZO and SCALEPACK (Otwinowski \& Minor, 1997), SIR2004 (Burla et al., 2005), PLATON (Spek, 2009) and JANA2006 (Petř́ček et al., 2014). Extinction correction: Becker \& Coppens (1974).

\section{Acknowledgements}

The author is grateful for the support of the Ministry of Education of the Czech Republic under Project NPU I-LO1603.

\section{References}

Becker, P. J. \& Coppens, P. (1974). Acta Cryst. A30, 129-147.

Benali-Cherif, N., Falek, W. \& Direm, A. (2009). Acta Cryst. E65, o3058-o3059.

Burla, M. C., Caliandro, R., Camalli, M., Carrozzini, B., Cascarano, G. L., De Caro, L., Giacovazzo, C., Polidori, G. \& Spagna, R. (2005). J. Appl. Cryst. 38, 381-388.

Etter, M. C., MacDonald, J. C. \& Bernstein, J. (1990). Acta Cryst. B46, 256-262.

Fábry, J., Dušek, M., Vaněk, P., Rafalovskyi, I., Hlinka, J. \& Urban, J. (2014). Acta Cryst. C70, 1153-1160.

Groom, C. R. \& Allen, F. H. (2014). Angew. Chem. Int. Ed. 53, 662671.

Nonius (1998). KappaCCD Server Software. Nonius BV, Delft, The Netherlands.

Otwinowski, Z. \& Minor, W. (1997). Methods in Enzymology, Vol. 276, Macromolecular Crystallography, Part A, edited by C. W. Carter Jr \& R. M. Sweet, pp. 307-326. New York: Academic Press.

Petř́ček, V., Dušek, M. \& Palatinus, L. (2014). Z. Kristallogr. 229, 345-352.

Spek, A. L. (2009). Acta Cryst. D65, 148-155. 


\section{supporting information}

Acta Cryst. (2016). E72, 509-511［https://doi.org/10.1107/S2056989016003923]

\section{Redetermination of cytosinium hydrogen maleate-cytosine (1/1) from the original data}

\section{Jan Fábry}

Computing details

Data collection: KappaCCD Server Software (Nonius, 1998); cell refinement: DENZO and SCALEPACK (Otwinowski \& Minor, 1997); data reduction: DENZO and SCALEPACK (Otwinowski \& Minor, 1997); program(s) used to solve structure: SIR2004 (Burla et al., 2005); program(s) used to refine structure: JANA2006 (Petř́ícke et al., 2014); molecular graphics: PLATON (Spek, 2009) and JANA2006 (Petříček et al., 2014); software used to prepare material for publication: JANA2006 (Petříček et al., 2014).

Cytosinium hydrogen maleate-cytosine (1/1)

Crystal data

$\mathrm{C}_{4} \mathrm{H}_{6} \mathrm{~N}_{3} \mathrm{O}^{+} \cdot \mathrm{C}_{4} \mathrm{H}_{3} \mathrm{O}_{4}^{-} \cdot \mathrm{C}_{4} \mathrm{H}_{5} \mathrm{~N}_{3} \mathrm{O}$

$M_{r}=338.29$

Monoclinic, $C 2 / c$

Hall symbol: $-\mathrm{C} 2 \mathrm{yc}$

$a=27.3226(5) \AA$

$b=7.3618(2) \AA$

$c=14.6742(4) \AA$

$\beta=93.905(1)^{\circ}$

$V=2944.77(13) \AA^{3}$

$Z=8$

\section{Data collection}

\section{Nonius KappaCCD} diffractometer

Radiation source: fine-focus sealed tube Graphite monochromator $\omega-\theta$ scans

3490 measured reflections

3474 independent reflections

Refinement

Refinement on $F^{2}$

$R[F>3 \sigma(F)]=0.038$

$w R(F)=0.093$

$S=1.85$

3474 reflections

246 parameters

2 restraints

33 constraints
$F(000)=1408$

$D_{\mathrm{x}}=1.526 \mathrm{Mg} \mathrm{m}^{-3}$

Mo $K \alpha$ radiation, $\lambda=0.71073 \AA$

Cell parameters from 3490 reflections

$\theta=2.8-28.0^{\circ}$

$\mu=0.13 \mathrm{~mm}^{-1}$

$T=298 \mathrm{~K}$

Prism, colourless

$0.3 \times 0.15 \times 0.1 \mathrm{~mm}$

2367 reflections with $I>3 \sigma(I)$

$R_{\text {int }}=0.043$

$\theta_{\text {max }}=28.0^{\circ}, \theta_{\min }=2.8^{\circ}$

$h=0 \rightarrow 35$

$k=0 \rightarrow 9$

$l=-19 \rightarrow 19$

$\mathrm{H}$ atoms treated by a mixture of independent and constrained refinement

Weighting scheme based on measured s.u.'s $w=$ $1 /\left(\sigma^{2}(I)+0.0004 I^{2}\right)$

$(\Delta / \sigma)_{\max }=0.022$

$\Delta \rho_{\max }=0.20 \mathrm{e} \AA^{-3}$

$\Delta \rho_{\min }=-0.20 \mathrm{e} \AA^{-3}$ 
Extinction correction: B-C type 1 Lorentzian isotropic (Becker \& Coppens, 1974)
Extinction coefficient: 21000 (5000)

\section{Special details}

Refinement. This part differs from the original article by Benali-Cherif et al. (2009). In the refinement, $\mathrm{F}^{2}>3 \sigma\left(\mathrm{F}^{2}\right)$ has been used as a criterion for observed diffractions.

The diffractions for which $\|\mathrm{Fo}|-| \mathrm{Fc}\|>10 \sigma(\mathrm{F})$ were discarded from the refinement. This refers to the diffractions $510 ;-91$ $1 ;-111 ;-821 ; 421 ;-202 ; 002 ;-312 ;-2008 ; 2228$.

Fractional atomic coordinates and isotropic or equivalent isotropic displacement parameters $\left(\AA^{2}\right)$

\begin{tabular}{|c|c|c|c|c|c|}
\hline & $x$ & $y$ & $z$ & $U_{\text {iso }} * / U_{\text {eq }}$ & Occ. $(<1)$ \\
\hline $\mathrm{O} 7 \mathrm{~b}$ & $0.33926(3)$ & $1.06385(11)$ & $0.29287(6)$ & $0.0445(3)$ & \\
\hline N1b & $0.40065(3)$ & $0.90018(14)$ & $0.23744(7)$ & 0.0400 & \\
\hline $\mathrm{H} 1 \mathrm{~b}$ & $0.4157(5)$ & $1.011(2)$ & $0.2197(9)$ & $0.048 *$ & \\
\hline $\mathrm{N} 3 \mathrm{~b}$ & $0.33591(3)$ & $0.75686(12)$ & $0.30318(7)$ & 0.0368 & \\
\hline $\mathrm{N} 8 \mathrm{~b}$ & $0.33297(5)$ & $0.44786(15)$ & $0.31476(9)$ & $0.0506(4)$ & \\
\hline H8b1 & $0.3454(5)$ & $0.338(2)$ & $0.3026(9)$ & $0.0607^{*}$ & \\
\hline H8b2 & $0.3002(6)$ & $0.458(2)$ & $0.3394(9)$ & $0.0607 *$ & \\
\hline $\mathrm{C} 2 \mathrm{~b}$ & $0.35770(4)$ & $0.91429(16)$ & $0.27860(8)$ & $0.0352(4)$ & \\
\hline $\mathrm{C} 4 \mathrm{~b}$ & $0.35664(4)$ & $0.59317(16)$ & $0.28928(8)$ & $0.0386(4)$ & \\
\hline $\mathrm{C} 5 \mathrm{~b}$ & $0.40187(4)$ & $0.58331(18)$ & 0.24835 (9) & $0.0443(4)$ & \\
\hline $\mathrm{H} 5 \mathrm{~b}$ & 0.416945 & 0.472174 & 0.23913 & $0.0531 *$ & \\
\hline $\mathrm{C} 6 \mathrm{~b}$ & $0.42233(5)$ & $0.73892(18)$ & $0.22326(9)$ & $0.0443(4)$ & \\
\hline $\mathrm{H} 6 \mathrm{~b}$ & 0.451959 & 0.735942 & 0.195619 & $0.0531 *$ & \\
\hline $\mathrm{O} 7 \mathrm{a}$ & $0.23772(3)$ & $0.47438(12)$ & $0.38018(7)$ & $0.0481(3)$ & \\
\hline N1a & $0.17576(3)$ & $0.63613(15)$ & $0.43512(7)$ & $0.0413(3)$ & \\
\hline H1a & $0.1595(4)$ & $0.528(2)$ & $0.4518(9)$ & $0.0496^{*}$ & \\
\hline N3a & 0.24303 & $0.78185(13)$ & $0.37815(7)$ & $0.0375(3)$ & \\
\hline N8a & $0.24808(5)$ & $1.09110(16)$ & $0.37611(9)$ & $0.0522(4)$ & \\
\hline H8a1 & $0.2767(6)$ & $1.0812(19)$ & $0.3501(10)$ & $0.0627^{*}$ & \\
\hline $\mathrm{H} 8 \mathrm{a} 2$ & $0.2363(5)$ & $1.200(2)$ & $0.3886(10)$ & $0.0627 *$ & \\
\hline $\mathrm{C} 2 \mathrm{a}$ & 0.21958 & $0.62341(16)$ & $0.39695(8)$ & $0.0364(4)$ & \\
\hline $\mathrm{C} 4 \mathrm{a}$ & $0.22340(4)$ & $0.94481(16)$ & $0.39665(8)$ & $0.0388(4)$ & \\
\hline $\mathrm{C} 5 \mathrm{a}$ & 0.17788 & $0.95478(18)$ & $0.43679(9)$ & $0.0439(4)$ & \\
\hline $\mathrm{H} 5 \mathrm{a}$ & 0.164021 & 1.066081 & 0.450387 & $0.0527^{*}$ & \\
\hline C6a & $0.15539(5)$ & $0.79794(18)$ & $0.45457(9)$ & $0.0447(4)$ & \\
\hline H6a & 0.125322 & 0.800462 & 0.480683 & $0.0536 *$ & \\
\hline $\mathrm{O} 1$ & 0.00024 & $0.51326(12)$ & $0.62974(6)$ & 0.0463 & \\
\hline $\mathrm{O} 2$ & $-0.05067(3)$ & $0.30108(13)$ & $0.67262(7)$ & 0.0560 & \\
\hline $\mathrm{O} 3$ & 0.07413 & $0.53082(11)$ & $0.54869(6)$ & 0.0433 & \\
\hline $\mathrm{H} 3$ & 0.0368 & $0.535(2)$ & $0.5874(9)$ & $0.0649 *$ & \\
\hline O4 & $0.12217(3)$ & $0.33900(14)$ & $0.48088(7)$ & 0.0555 & \\
\hline $\mathrm{C} 1$ & $0.08603(4)$ & $0.37076(18)$ & $0.52453(8)$ & $0.0394(4)$ & \\
\hline $\mathrm{C} 2$ & $0.05595(5)$ & 0.21144 (19) & $0.54864(10)$ & $0.0512(5)$ & \\
\hline H1 & 0.067397 & 0.100138 & 0.528962 & $0.0614^{*}$ & \\
\hline $\mathrm{C} 3$ & $0.01546(5)$ & 0.20204 (19) & $0.59362(10)$ & $0.0523(5)$ & \\
\hline $\mathrm{H} 2$ & 0.003281 & 0.085236 & 0.600501 & $0.0628 *$ & \\
\hline $\mathrm{C} 4$ & -0.01355 (4) & $0.34770(18)$ & $0.63476(8)$ & $0.0415(4)$ & \\
\hline
\end{tabular}




\begin{tabular}{llllll}
$\mathrm{H} 1 \mathrm{n} 3 \mathrm{~b}$ & $0.3079(5)$ & $0.763(3)$ & $0.3267(14)$ & $0.0442^{*}$ & $0.554(16)$ \\
$\mathrm{H} 1 \mathrm{n} 3 \mathrm{a}$ & $0.2718(6)$ & $0.783(4)$ & $0.3556(18)$ & $0.045^{*}$ & $0.446(16)$ \\
\hline
\end{tabular}

Atomic displacement parameters $\left(\AA^{2}\right)$

\begin{tabular}{lllllll}
\hline & $U^{11}$ & $U^{22}$ & $U^{33}$ & $U^{12}$ & $U^{13}$ & $U^{23}$ \\
\hline O7b & $0.0462(5)$ & $0.0256(5)$ & $0.0628(6)$ & $0.0015(3)$ & $0.0110(4)$ & $-0.0015(4)$ \\
N1b & $0.0413(6)$ & $0.0342(6)$ & $0.0454(6)$ & $-0.0014(4)$ & $0.0100(5)$ & $-0.0016(5)$ \\
N3b & $0.0375(5)$ & $0.0249(6)$ & $0.0486(6)$ & $0.0009(4)$ & $0.0073(4)$ & $-0.0018(4)$ \\
N8b & $0.0551(7)$ & $0.0278(6)$ & $0.0705(8)$ & $0.0025(5)$ & $0.0171(6)$ & $0.0000(5)$ \\
C2b & $0.0374(6)$ & $0.0291(7)$ & $0.0388(7)$ & $0.0009(5)$ & $0.0007(5)$ & $-0.0022(5)$ \\
C4b & $0.0450(6)$ & $0.0285(7)$ & $0.0420(7)$ & $0.0019(5)$ & $0.0002(5)$ & $-0.0021(5)$ \\
C5b & $0.0452(7)$ & $0.0368(8)$ & $0.0516(8)$ & $0.0105(5)$ & $0.0093(6)$ & $-0.0029(6)$ \\
C6b & $0.0425(6)$ & $0.0435(8)$ & $0.0476(8)$ & $0.0062(5)$ & $0.0093(6)$ & $-0.0040(6)$ \\
O7a & $0.0468(5)$ & $0.0246(5)$ & $0.0740(6)$ & $0.0004(4)$ & $0.0134(4)$ & $-0.0008(4)$ \\
N1a & $0.0382(5)$ & $0.0335(6)$ & $0.0532(6)$ & $-0.0044(4)$ & $0.0100(5)$ & $-0.0018(5)$ \\
N3a & $0.0354(5)$ & $0.0246(5)$ & $0.0531(6)$ & $0.0008(4)$ & $0.0079(4)$ & $-0.0001(4)$ \\
N8a & $0.0515(6)$ & $0.0263(6)$ & $0.0806(9)$ & $0.0013(5)$ & $0.0171(6)$ & $0.0004(6)$ \\
C2a & $0.0374(6)$ & $0.0269(7)$ & $0.0448(7)$ & $0.0008(5)$ & $0.0024(5)$ & $0.0000(5)$ \\
C4a & $0.0400(6)$ & $0.0296(7)$ & $0.0467(7)$ & $0.0024(5)$ & $0.0009(5)$ & $-0.0017(5)$ \\
C5a & $0.0421(7)$ & $0.0341(7)$ & $0.0560(8)$ & $0.0085(5)$ & $0.0059(6)$ & $-0.0059(6)$ \\
C6a & $0.0375(6)$ & $0.0457(8)$ & $0.0514(8)$ & $0.0032(5)$ & $0.0073(6)$ & $-0.0065(6)$ \\
O1 & $0.0411(5)$ & $0.0411(6)$ & $0.0582(6)$ & $-0.0016(4)$ & $0.0155(4)$ & $-0.0041(4)$ \\
O2 & $0.0471(5)$ & $0.0532(6)$ & $0.0703(7)$ & $-0.0059(4)$ & $0.0234(5)$ & $0.0033(5)$ \\
O3 & $0.0426(5)$ & $0.0365(5)$ & $0.0521(5)$ & $-0.0035(4)$ & $0.0137(4)$ & $-0.0025(4)$ \\
O4 & $0.0547(5)$ & $0.0476(6)$ & $0.0677(6)$ & $-0.0001(4)$ & $0.0288(5)$ & $-0.0052(5)$ \\
C1 & $0.0402(6)$ & $0.0388(7)$ & $0.0398(7)$ & $0.0003(5)$ & $0.0070(5)$ & $-0.0002(6)$ \\
C2 & $0.0545(8)$ & $0.0338(8)$ & $0.0675(9)$ & $0.0020(6)$ & $0.0202(7)$ & $-0.0028(6)$ \\
C3 & $0.0544(8)$ & $0.0332(8)$ & $0.0714(10)$ & $-0.0045(6)$ & $0.0185(7)$ & $0.0033(7)$ \\
C4 & $0.0383(6)$ & $0.0417(8)$ & $0.0449(7)$ & $-0.0028(5)$ & $0.0059(5)$ & $0.0025(6)$ \\
& & & & & &
\end{tabular}

Geometric parameters $\left(\AA,{ }^{\circ}\right)$

\begin{tabular}{llll}
\hline $\mathrm{O} 7 \mathrm{~b}-\mathrm{C} 2 \mathrm{~b}$ & $1.2345(14)$ & $\mathrm{N} 3 \mathrm{a}-\mathrm{H} 1 \mathrm{n} 3 \mathrm{a}$ & $0.873(18)$ \\
$\mathrm{N} 1 \mathrm{~b}-\mathrm{H} 1 \mathrm{~b}$ & $0.956(15)$ & $\mathrm{N} 8 \mathrm{a}-\mathrm{H} 8 \mathrm{a} 1$ & $0.897(16)$ \\
$\mathrm{N} 1 \mathrm{~b}-\mathrm{C} 2 \mathrm{~b}$ & $1.3600(15)$ & N8a-H8a2 & $0.885(17)$ \\
$\mathrm{N} 1 \mathrm{~b}-\mathrm{C} 6 \mathrm{~b}$ & $1.3492(17)$ & $\mathrm{N} 8 \mathrm{a}-\mathrm{C} 4 \mathrm{a}$ & $1.3165(17)$ \\
$\mathrm{N} 3 \mathrm{~b}-\mathrm{C} 2 \mathrm{~b}$ & $1.3627(15)$ & $\mathrm{H} 8 \mathrm{a} 1-\mathrm{H} 8 \mathrm{a} 2$ & $1.54(2)$ \\
$\mathrm{N} 3 \mathrm{~b}-\mathrm{C} 4 \mathrm{~b}$ & $1.3528(15)$ & $\mathrm{C} 4 \mathrm{a}-\mathrm{C} 5 \mathrm{a}$ & $1.4137(17)$ \\
$\mathrm{N} 3 \mathrm{~b}-\mathrm{H} 1 \mathrm{n} 3 \mathrm{~b}$ & $0.861(16)$ & $\mathrm{C} 5 \mathrm{a}-\mathrm{H} 5 \mathrm{a}$ & 0.93 \\
$\mathrm{~N} 8 \mathrm{~b}-\mathrm{H} 8 \mathrm{~b} 1$ & $0.900(17)$ & $\mathrm{C} 5 \mathrm{a}-\mathrm{C} 6 \mathrm{a}$ & $1.3420(19)$ \\
$\mathrm{N} 8 \mathrm{~b}-\mathrm{H} 8 \mathrm{~b} 2$ & $0.992(15)$ & $\mathrm{C} 6 \mathrm{a}-\mathrm{H} 6 \mathrm{a}$ & 0.93 \\
$\mathrm{~N} 8 \mathrm{~b}-\mathrm{C} 4 \mathrm{~b}$ & $1.3174(17)$ & $\mathrm{O} 1-\mathrm{H} 3$ & $1.223(14)$ \\
$\mathrm{H} 8 \mathrm{~b} 1-\mathrm{H} 8 \mathrm{~b} 2$ & $1.64(2)$ & $\mathrm{O} 1-\mathrm{C} 4$ & $1.2793(16)$ \\
$\mathrm{C} 4 \mathrm{~b}-\mathrm{C} 5 \mathrm{~b}$ & $1.4123(17)$ & O2-C4 & $1.2376(15)$ \\
$\mathrm{C} 5 \mathrm{~b}-\mathrm{H} 5 \mathrm{~b}$ & 0.93 & $\mathrm{O} 3-\mathrm{H} 3$ & $1.201(14)$ \\
$\mathrm{C} 5 \mathrm{~b}-\mathrm{C} 6 \mathrm{~b}$ & $1.3373(18)$ & O3-C1 & $1.2789(15)$ \\
$\mathrm{C} 6 \mathrm{~b}-\mathrm{H} 6 \mathrm{~b}$ & 0.93 & O4-C1 & $1.2354(15)$
\end{tabular}




\begin{tabular}{|c|c|c|c|}
\hline $\mathrm{O} 7 \mathrm{a}-\mathrm{C} 2 \mathrm{a}$ & $1.2356(14)$ & $\mathrm{C} 1-\mathrm{C} 2$ & $1.4886(19)$ \\
\hline $\mathrm{N} 1 \mathrm{a}-\mathrm{H} 1 \mathrm{a}$ & $0.952(14)$ & $\mathrm{C} 2-\mathrm{H} 1$ & 0.93 \\
\hline $\mathrm{N} 1 \mathrm{a}-\mathrm{C} 2 \mathrm{a}$ & $1.3591(15)$ & $\mathrm{C} 2-\mathrm{C} 3$ & $1.328(2)$ \\
\hline $\mathrm{N} 1 \mathrm{a}-\mathrm{C} 6 \mathrm{a}$ & $1.3535(17)$ & $\mathrm{C} 3-\mathrm{H} 2$ & 0.93 \\
\hline $\mathrm{N} 3 \mathrm{a}-\mathrm{C} 2 \mathrm{a}$ & $1.3679(15)$ & $\mathrm{C} 3-\mathrm{C} 4$ & $1.4859(19)$ \\
\hline $\mathrm{N} 3 \mathrm{a}-\mathrm{C} 4 \mathrm{a}$ & $1.3493(15)$ & $\mathrm{H} 1 \mathrm{n} 3 \mathrm{~b}-\mathrm{H} 1 \mathrm{n} 3 \mathrm{a}$ & $1.11(2)$ \\
\hline $\mathrm{H} 1 \mathrm{~b}-\mathrm{N} 1 \mathrm{~b}-\mathrm{C} 2 \mathrm{~b}$ & $117.2(8)$ & $\mathrm{H} 8 \mathrm{a} 2-\mathrm{N} 8 \mathrm{a}-\mathrm{C} 4 \mathrm{a}$ & $119.4(10)$ \\
\hline $\mathrm{H} 1 \mathrm{~b}-\mathrm{N} 1 \mathrm{~b}-\mathrm{C} 6 \mathrm{~b}$ & $120.3(8)$ & $\mathrm{O} 7 \mathrm{a}-\mathrm{C} 2 \mathrm{a}-\mathrm{N} 1 \mathrm{a}$ & $121.32(11)$ \\
\hline $\mathrm{C} 2 \mathrm{~b}-\mathrm{N} 1 \mathrm{~b}-\mathrm{C} 6 \mathrm{~b}$ & $122.48(11)$ & $\mathrm{O} 7 \mathrm{a}-\mathrm{C} 2 \mathrm{a}-\mathrm{N} 3 \mathrm{a}$ & $121.14(10)$ \\
\hline $\mathrm{C} 2 \mathrm{~b}-\mathrm{N} 3 \mathrm{~b}-\mathrm{C} 4 \mathrm{~b}$ & $121.52(10)$ & $\mathrm{N} 1 \mathrm{a}-\mathrm{C} 2 \mathrm{a}-\mathrm{N} 3 \mathrm{a}$ & $117.53(10)$ \\
\hline $\mathrm{C} 2 \mathrm{~b}-\mathrm{N} 3 \mathrm{~b}-\mathrm{H} 1 \mathrm{n} 3 \mathrm{~b}$ & $118.7(15)$ & $\mathrm{N} 3 \mathrm{a}-\mathrm{C} 4 \mathrm{a}-\mathrm{N} 8 \mathrm{a}$ & $117.68(11)$ \\
\hline $\mathrm{C} 4 \mathrm{~b}-\mathrm{N} 3 \mathrm{~b}-\mathrm{H} 1 \mathrm{n} 3 \mathrm{~b}$ & $119.8(15)$ & $\mathrm{N} 3 \mathrm{a}-\mathrm{C} 4 \mathrm{a}-\mathrm{C} 5 \mathrm{a}$ & $120.20(11)$ \\
\hline $\mathrm{H} 8 \mathrm{~b} 1-\mathrm{N} 8 \mathrm{~b}-\mathrm{H} 8 \mathrm{~b} 2$ & $120.1(13)$ & $\mathrm{N} 8 \mathrm{a}-\mathrm{C} 4 \mathrm{a}-\mathrm{C} 5 \mathrm{a}$ & $122.12(12)$ \\
\hline $\mathrm{H} 8 \mathrm{~b} 1-\mathrm{N} 8 \mathrm{~b}-\mathrm{C} 4 \mathrm{~b}$ & $118.2(9)$ & $\mathrm{C} 4 \mathrm{a}-\mathrm{C} 5 \mathrm{a}-\mathrm{H} 5 \mathrm{a}$ & 121.18 \\
\hline $\mathrm{H} 8 \mathrm{~b} 2-\mathrm{N} 8 \mathrm{~b}-\mathrm{C} 4 \mathrm{~b}$ & $121.2(9)$ & $\mathrm{C} 4 \mathrm{a}-\mathrm{C} 5 \mathrm{a}-\mathrm{C} 6 \mathrm{a}$ & $117.64(12)$ \\
\hline $\mathrm{O} 7 \mathrm{~b}-\mathrm{C} 2 \mathrm{~b}-\mathrm{N} 1 \mathrm{~b}$ & $121.19(11)$ & $\mathrm{H} 5 \mathrm{a}-\mathrm{C} 5 \mathrm{a}-\mathrm{C} 6 \mathrm{a}$ & 121.18 \\
\hline $\mathrm{O} 7 \mathrm{~b}-\mathrm{C} 2 \mathrm{~b}-\mathrm{N} 3 \mathrm{~b}$ & $121.54(10)$ & $\mathrm{N} 1 \mathrm{a}-\mathrm{C} 6 \mathrm{a}-\mathrm{C} 5 \mathrm{a}$ & $121.06(12)$ \\
\hline $\mathrm{N} 1 \mathrm{~b}-\mathrm{C} 2 \mathrm{~b}-\mathrm{N} 3 \mathrm{~b}$ & $117.28(10)$ & $\mathrm{N} 1 \mathrm{a}-\mathrm{C} 6 \mathrm{a}-\mathrm{H} 6 \mathrm{a}$ & 119.47 \\
\hline $\mathrm{N} 3 \mathrm{~b}-\mathrm{C} 4 \mathrm{~b}-\mathrm{N} 8 \mathrm{~b}$ & $117.51(11)$ & $\mathrm{C} 5 \mathrm{a}-\mathrm{C} 6 \mathrm{a}-\mathrm{H} 6 \mathrm{a}$ & 119.47 \\
\hline $\mathrm{N} 3 \mathrm{~b}-\mathrm{C} 4 \mathrm{~b}-\mathrm{C} 5 \mathrm{~b}$ & $119.83(11)$ & $\mathrm{H} 3-\mathrm{O} 1-\mathrm{C} 4$ & $114.1(8)$ \\
\hline $\mathrm{N} 8 \mathrm{~b}-\mathrm{C} 4 \mathrm{~b}-\mathrm{C} 5 \mathrm{~b}$ & $122.67(11)$ & $\mathrm{H} 3-\mathrm{O} 3-\mathrm{C} 1$ & $113.3(8)$ \\
\hline $\mathrm{C} 4 \mathrm{~b}-\mathrm{C} 5 \mathrm{~b}-\mathrm{H} 5 \mathrm{~b}$ & 121.07 & $\mathrm{O} 1-\mathrm{H} 3-\mathrm{O} 3$ & $170.6(15)$ \\
\hline $\mathrm{C} 4 \mathrm{~b}-\mathrm{C} 5 \mathrm{~b}-\mathrm{C} 6 \mathrm{~b}$ & $117.86(12)$ & $\mathrm{O} 3-\mathrm{C} 1-\mathrm{O} 4$ & $123.05(12)$ \\
\hline $\mathrm{H} 5 \mathrm{~b}-\mathrm{C} 5 \mathrm{~b}-\mathrm{C} 6 \mathrm{~b}$ & 121.07 & $\mathrm{O} 3-\mathrm{C} 1-\mathrm{C} 2$ & $120.35(11)$ \\
\hline $\mathrm{N} 1 \mathrm{~b}-\mathrm{C} 6 \mathrm{~b}-\mathrm{C} 5 \mathrm{~b}$ & $121.00(12)$ & $\mathrm{O} 4-\mathrm{C} 1-\mathrm{C} 2$ & $116.60(12)$ \\
\hline $\mathrm{N} 1 \mathrm{~b}-\mathrm{C} 6 \mathrm{~b}-\mathrm{H} 6 \mathrm{~b}$ & 119.5 & $\mathrm{C} 1-\mathrm{C} 2-\mathrm{H} 1$ & 114.68 \\
\hline $\mathrm{C} 5 \mathrm{~b}-\mathrm{C} 6 \mathrm{~b}-\mathrm{H} 6 \mathrm{~b}$ & 119.5 & $\mathrm{C} 1-\mathrm{C} 2-\mathrm{C} 3$ & $130.64(13)$ \\
\hline $\mathrm{H} 1 \mathrm{a}-\mathrm{N} 1 \mathrm{a}-\mathrm{C} 2 \mathrm{a}$ & $119.2(8)$ & $\mathrm{H} 1-\mathrm{C} 2-\mathrm{C} 3$ & 114.68 \\
\hline $\mathrm{H} 1 \mathrm{a}-\mathrm{N} 1 \mathrm{a}-\mathrm{C} 6 \mathrm{a}$ & $118.4(8)$ & $\mathrm{C} 2-\mathrm{C} 3-\mathrm{H} 2$ & 114.74 \\
\hline $\mathrm{C} 2 \mathrm{a}-\mathrm{N} 1 \mathrm{a}-\mathrm{C} 6 \mathrm{a}$ & $122.28(11)$ & $\mathrm{C} 2-\mathrm{C} 3-\mathrm{C} 4$ & $130.53(13)$ \\
\hline $\mathrm{C} 2 \mathrm{a}-\mathrm{N} 3 \mathrm{a}-\mathrm{C} 4 \mathrm{a}$ & $121.30(10)$ & $\mathrm{H} 2-\mathrm{C} 3-\mathrm{C} 4$ & 114.74 \\
\hline $\mathrm{C} 2 \mathrm{a}-\mathrm{N} 3 \mathrm{a}-\mathrm{H} 1 \mathrm{n} 3 \mathrm{a}$ & $121.9(18)$ & $\mathrm{O} 1-\mathrm{C} 4-\mathrm{O} 2$ & $122.93(12)$ \\
\hline $\mathrm{C} 4 \mathrm{a}-\mathrm{N} 3 \mathrm{a}-\mathrm{H} 1 \mathrm{n} 3 \mathrm{a}$ & $116.8(18)$ & $\mathrm{O} 1-\mathrm{C} 4-\mathrm{C} 3$ & $119.78(11)$ \\
\hline $\mathrm{H} 8 \mathrm{a} 1-\mathrm{N} 8 \mathrm{a}-\mathrm{H} 8 \mathrm{a} 2$ & $120.2(14)$ & $\mathrm{O} 2-\mathrm{C} 4-\mathrm{C} 3$ & $117.28(12)$ \\
\hline $\mathrm{H} 8 \mathrm{a} 1-\mathrm{N} 8 \mathrm{a}-\mathrm{C} 4 \mathrm{a}$ & $120.4(9)$ & & \\
\hline
\end{tabular}

Hydrogen-bond geometry $\left(A,{ }^{\circ}\right)$

\begin{tabular}{lllll}
\hline$D-\mathrm{H}^{\cdots} A$ & $D-\mathrm{H}$ & $\mathrm{H} \cdots A$ & $D \cdots A$ & $D-\mathrm{H}^{\cdots} A$ \\
\hline $\mathrm{N} 1 b-\mathrm{H} 1 b \cdots \mathrm{O} 2^{\mathrm{i}}$ & $0.956(15)$ & $1.824(15)$ & $2.7718(14)$ & $170.8(13)$ \\
$\mathrm{N} 8 b-\mathrm{H} 8 b 1 \cdots \mathrm{O} 7 b^{\mathrm{ii}}$ & $0.900(17)$ & $2.030(18)$ & $2.8517(14)$ & $151.2(13)$ \\
$\mathrm{N} 8 b-\mathrm{H} 8 b 2 \cdots \mathrm{O} 7 a$ & $0.992(15)$ & $1.850(15)$ & $2.8411(15)$ & $177.4(12)$ \\
$\mathrm{C} 5 b-\mathrm{H} 5 b \cdots \mathrm{O} 2^{\mathrm{iii}}$ & 0.93 & 2.43 & $3.3347(16)$ & 164.60 \\
$\mathrm{~N} 1 a-\mathrm{H} 1 a \cdots \mathrm{O} 4$ & $0.952(14)$ & $1.793(14)$ & $2.7411(14)$ & $173.2(12)$ \\
$\mathrm{N} 8 a-\mathrm{H} 8 a 1 \cdots \mathrm{O} 7 b$ & $0.897(16)$ & $1.959(16)$ & $2.8555(15)$ & $179.0(13)$ \\
$\mathrm{N} 8 a-\mathrm{H} 8 a 2 \cdots \mathrm{O} 7 a^{\mathrm{iv}}$ & $0.885(17)$ & $2.028(18)$ & $2.8368(15)$ & $151.5(14)$ \\
$\mathrm{C} 5 a-\mathrm{H} 5 a \cdots \mathrm{O} 44^{\text {iv }}$ & 0.93 & 2.37 & $3.2970(16)$ & 175.15
\end{tabular}


supporting information

\begin{tabular}{lllll}
$\mathrm{O} 1-\mathrm{H} 3 \cdots \mathrm{O} 3$ & $1.223(14)$ & $1.201(14)$ & $2.4155(12)$ & $170.6(15)$ \\
$\mathrm{O} 1-\mathrm{H} 3 \cdots \mathrm{C} 1$ & $1.223(14)$ & $2.071(15)$ & $3.0775(15)$ & $136.7(11)$ \\
$\mathrm{O} 3-\mathrm{H} 3 \cdots \mathrm{O} 1$ & $1.201(14)$ & $1.223(14)$ & $2.4155(12)$ & $170.6(15)$ \\
$\mathrm{O} 3-\mathrm{H} 3 \cdots \mathrm{C} 4$ & $1.201(14)$ & $2.100(15)$ & $3.0927(15)$ & $137.4(12)$ \\
$\mathrm{N} 3 b-\mathrm{H} 1 n 3 b \cdots \mathrm{N} 3 a$ & $0.861(16)$ & $1.979(16)$ & $2.8398(14)$ & $178(2)$ \\
$\mathrm{N} 3 a-\mathrm{H} 1 n 3 a \cdots \mathrm{N} 3 b$ & $0.873(18)$ & $1.970(18)$ & $2.8398(14)$ & $174(3)$ \\
\hline
\end{tabular}

Symmetry codes: (i) $x+1 / 2,-y+3 / 2, z-1 / 2$; (ii) $x, y-1, z$; (iii) $x+1 / 2,-y+1 / 2, z-1 / 2$; (iv) $x, y+1, z$. 